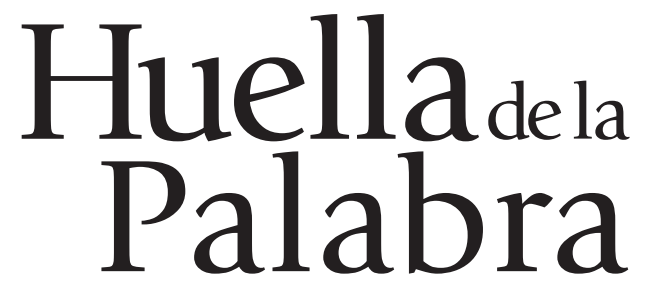

Revista de Investigación Educativa

Universidad La Salle Pachuca

lahuelladelapalabra@lasallep.edu.mx

Teléfono: 01(771) 7170213 ext. 1406

Fax: 01(771) 7170309

ISSN: En trámite

México

Camarillo Madrid Elsa María

Vázquez Monter Silvia Carolina

Influencia de los estilos parentales en la autoestima de niños de 9 a 12 años

Huella de la Palabra, año 2015, número 9

Universidad La Salle Pachuca

pp. 33-42 



\section{Influencia de los estilos parentales en la autoestima de niños de 9 a 12 años}

Camarillo Madrid Elsa María ${ }^{1}$

Vázquez Monter Silvia Carolina

\section{Resumen}

La presente investigación aborda las consecuencias que los estilos parentales tienen en la autoestima, que es una de las variables más importante para la satisfacción personal de los seres humanos. Durante la realización de este estudio, se tomó como objetivo identificar la correlación existente entre los estilos parentales ejercidos por las madres y la autoestima desarrollada en niños de 9 a 12 años de edad, a través de su evaluación con el Cuestionario para la Evaluación de Autoestima en Educación Primaria A-EP (Ramos, Giménez, Muñoz-Adell y Lapaz, 2003) y de los estilos parentales con la Escala de Viñetas de Estilos Parentales (EVEP) (Vázquez, 2013), para determinar qué estilos parentales impulsan o detienen el desarrollo de una alta autoestima en los niños.

Los resultados indican una correlación significativa sobre la relación que existe entre el estilo parental ejercido y la autoestima manifestada, siendo el estilo democrático el primero y el segundo lugar el permisivo, los cuales influyen en niveles de autoestima más alta en los niños.

Palabras clave: Estilos parentales, autoestima, autoconfianza, niñez.

\begin{abstract}
This research addresses the consequences of parenting styles have on self-esteem, which is one of the most important personal satisfaction of human beings variables. While conducting this study and was taken as objective to identify the correlation between parenting
\end{abstract}

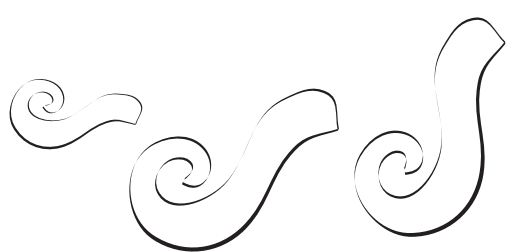

styles exercised by mothers and selfdeveloped in children 9 to 12 years of age, through self-assessment Questionnaire for evaluation of Self-Esteem in Elementary Education a-EP (Ramos, Giménez, Muñoz-Adell and Lapaz, 2003) and parenting styles with Scale styles parenting Bullets (EVEP) (Vázquez, 2013), to determine which parenting styles contributes or stop the development of high self-esteem in children. The results show a significant corelation between the relationship between parenting style and self-esteem manifested in democratic and permisive style causing higher self-esteem levels in the children.

Keywords: Parenting styles, self-esteem, selfconfidence, childhood.

\section{Introducción}

La autoestima se ha convertido durante los últimos 30 años en uno de los tópicos de mayor interés, los estudios realizados durante ese tiempo han arrojado aportaciones que se consideran clásicas. Diferentes modelos explicativos de la autoestima se han cimentado en estas investigaciones que les anteceden. Diversos autores como William James y Charles Cooley realizaron trabajos donde se denotó el interés en este tema desde principios de siglo, pero no es hasta mediados de los años 70 cuando las investigaciones comienzan a volverse sistemáticas y de tipo empírico, lo cual da un mayor soporte y respaldo para la comprensión de este concepto (Soto, 2012).

Es muy importante considerar la autoestima

1 elsa_camad91@hotmail.es, Licenciatura en Psicología

$$
\text { 20) } 35
$$




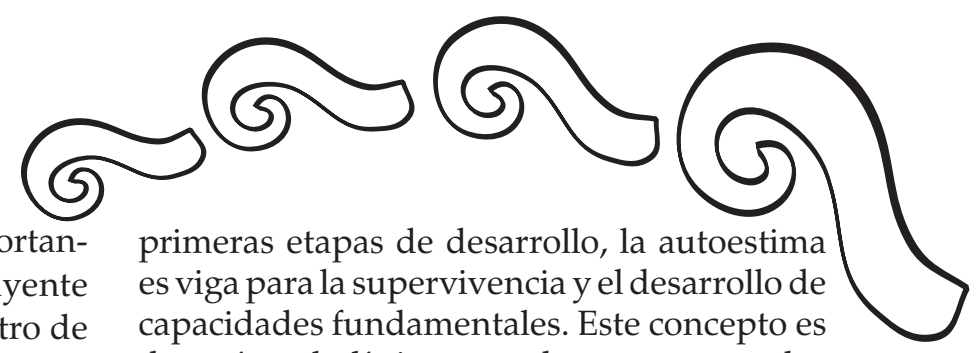

en general como un factor de gran importancia en las personas, ya que ésta es influyente para el desempeño de las personas dentro de las actividades académicas o desenvolvimiento laboral. Existen autores que describen la autoestima como el vestigio del alma, lo cual sería proporcional a lo que le da al ser humano dignidad. La evolución del yo, es lo que también le da cabida al desarrollo de la autoestima, sin autoestima no es posible una autorrealización (Contreras y López, 2010).

Una de las definiciones puntuales más citadas por los expertos es la de Mézerville (2004, p. 25 en Pereira, 2007).

“La autoestima está configurada por factores tanto internos como externos. Se entiende por factores internos, aquellos que radican o son creados por el individuoideas, creencias, prácticas o conductas. Así mismo por factores externos, los factores del entorno: los mensajes transmitidos verbal o no verbalmente, o las experiencias suscitadas por los padres, los educadores, las personas significativas, las organizaciones y la cultura."

Con respecto al punto anterior, la autoestima es básica para la satisfacción vital del ser humano ya que ésta es el cimiento para los planes y por lo tanto para un futuro, ya que gracias a ella se proyecta la seguridad que se tiene en sí mismo. Sin embargo esta relación no sería de ninguna forma homogénea si no se tomara en cuenta que cada persona desarrolla una autoestima paralela con los rasgos de la cultura en la cual crezca, la autoestima es una variable más fácil de observar en culturas individualistas y no es difícil asumir que esto se debe a que el interés está centrado en la persona y se ofrece una mayor calidad de servicios emocionales (San Martín y Barra, 2013).

De modo que, la autoestima y la percepción del sujeto están destinadas a marcar de forma significativa la manera cómo se desarrollan las enfermedades mentales en el sujeto. Desde el momento de la concepción, pasando por las de carácter holístico y ayuda como generador del fin último, el bienestar personal (Vera, Roselló, Toro-Alfonso, 2010).

Del mismo modo, Rosenberg (1965, en González y Gimeno, 2013) es uno de los pioneros en el estudio de la autoestima y quien crea una de las escalas más utilizadas por profesionales interesados en este tema; él considera que la escala debe de considerarse unidimensional. Sin embargo, Shavelson, Hubner y Stanton (1976) difieren de esta opinión y aportan que la autoestima tiene una estructura multidimensional ya que conforme la persona se desarrolla, ésta es capaz de identificar más dimensiones que se relacionan con la autoestima y es capaz también de darle más peso a unas que a otras (Rodrigues, Veiga y Fuentes, 2013).

Como es sabido, durante el desarrollo de la autoestima hay procesos que se deben consolidar de primera instancia como lo es la relación que se forja con los padres, el autocontrol personal, la conducta que se desempeña y el cómo y porqué de la autoaceptación, esto en el futuro del sujeto le facilitará crear el sustento de su identidad. Otro dato importante a tomar en cuenta es que el desarrollo es también dependiente de la cultura en la que crezca el sujeto (Contreras y López, 2009).

De manera que los traumas, pérdidas, desarreglos emocionales y otras circunstancias pueden afectar la vida de una persona y por lo tanto su autoestima. Es de esperarse que si no se resuelven estos conflictos emocionales, será muy probable que se desarrollen problemas a nivel de salud mental en el futuro del sujeto. Por esto se infiere que en cuanto más se trabajen los traumas de manera positiva en el autoestima será menos probable que en el futuro se presente una desintegración de su personalidad (Martínez, Rosete y de los Ríos, 2007). 


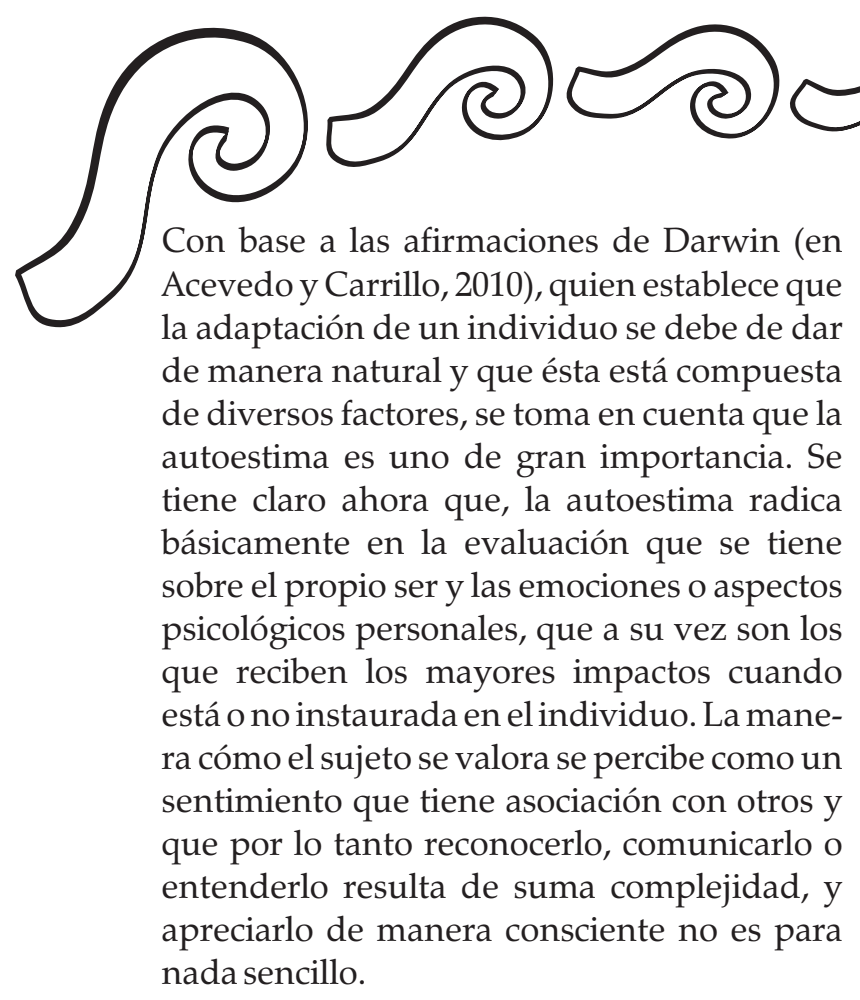

Es importante mencionar que las personas con una autoestima elevada o saludable, interpretan las experiencias que llegan a ser negativas de un modo más funcional, esto quiere decir que las afrontan y aprenden de ellas, lo cual hace fácil la retroalimentación y la utilización de las nuevas habilidades adquiridas para la resolución de otros problemas, generan atribuciones de mayor control, se muestran mucho más persistentes en las tareas que les resultan complicadas; lo que pasa con las personas de autoestima baja es completamente lo contrario (Naranjo y Caño, 2012).

Se puede decir que la autoestima es una especie de amortiguador personal que se encuentra en el interior de la persona y opera de distintas maneras, una de ellas es para disminuir el impacto que se podría causar ante las experiencias negativas que se viven constantemente. Quienes cuenten con mayores niveles de autoestima estarán menos propensos al impacto y es muy posible que experimenten una mayor satisfacción en distintos campos de su vida y pasa lo contrario con quienes cuentan con un bajo nivel de autoestima (San Martín y Barra, 2013).
Con relación a los estilos parentales, las investigaciones han mostrado que lo que las personas hacen o la manera cómo se comportan depende en gran medida de los estilos parentales que hayan usado sus padres. Es por esto que sabiendo que los padres son las primeras figuras de quienes se aprende de forma directa, entonces se puede inferir que la forma en la que ellos se comporten determina el desarrollo por lo menos en las primeras etapas de sus hijos (Galaz, Cortés y Góngora, 2009).

Las conductas específicas que llevan a cabo las madres y los padres durante en desarrollo de sus hijos, es conocido como: prácticas parentales, estilos de crianza, estilos de sociabilización parental o estilos parentales. Todos estos términos son equivalentes, pero para esta investigación el último de los mencionados será el que se utilizará de mayormente. Estas conductas llevan a un fin general, que es el poder proveer de enseñanza, cuidado y protección a los hijos. Dichas prácticas son básicamente planificaciones estratégicas que los padres diseñan consciente o inconscientemente para la crianza del menor (Moral, 2013). Estas prácticas son indirectas, lo que quiere decir que se instauran de forma inconsciente en la dinámica familiar y en la relación padrehijo con gestos, tonos de voz, reacciones espontaneas, repertorio verbal, etc. (Andrade y Morales, 2009).

Darling y Steinberg (1993) argumentan que el estilo parental que la persona desarrolla hacia su hijo depende en gran medida de la forma de ser de los padres que éste(a) tuvo en su infancia, lo cual quiere decir que la forma cómo se relacione un hijo con sus padres, será similar a la relación que sus padres llevaron a cabo con sus abuelos. A esto se le puede añadir que los padres les comunican a los hijos configuraciones en conjunto y éstas crean en la familia cierto tipo de ambiente emocional, dicho ambiente puede variar constantemente. Se considera que los estilos parentales son la raíz del aprendizaje, de la forma cómo los hijos se comportarán y el cómo establecerán vínculos emocionales (Climent, 2009). 


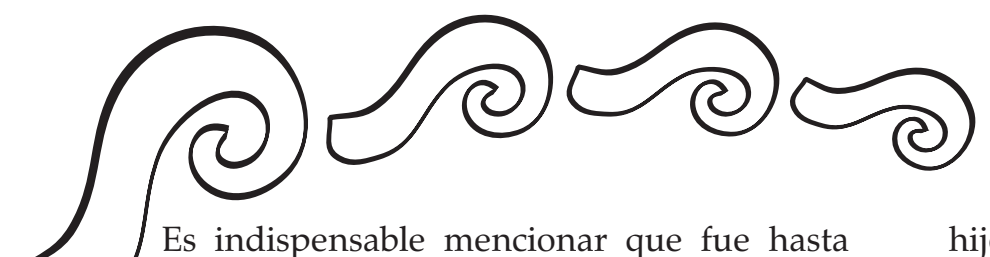

Baurmind (1966, en Andrade y Morales, 2099)

cuando se desarrollan términos específicos para el estilo parental. Este autor propone de forma inicial tres estilos: autoritarios, democráticos y permisivos. Posteriormente, esta autora amplía sus estilos a cuatro: democráti$\mathrm{Co}$, autoritario, permisivo y negligente. Esta última clasificación es apoyada por estudios realizados por Darling y Steinberg en una revisión teórica en 1993 (Torio, Peña y Rodríguez, 2008).

Asimismo, es de suma importancia aclarar los términos que pueden ser confundidos dentro del juego de palabras que se realiza en la investigación de los estilos parentales, por eso se debe puntualizar que existen definiciones que son sinónimas, tales como: autoritativo, autorizativo o democrático, asimismo permisivo e indulgente son palabras sinónimas, por último se aclara que negligente e indiferente deben de tomarse como palabras que contienen el mismo significado en la definición de los estilos parentales. Durante esta investigación serán empleados los términos: autoritario, democrático, permisivo y negligente.

Es por tanto que se debe tomar en cuenta la importancia de las acciones y comportamiento de cada padre como agente determinante del desarrollo del hijo. A continuación se desglosan de forma más amplia las tipologías de los estilos parentales antes mencionadas por Baurmind y confirmadas por Darling y Steinberg (en Vallejo, Osorno, y Mazadiego, 2008), los cuales consideran cuatro tipos de estilos parentales:

a) Estilo autoritario: se adjudica a los padres cuyo nivel de involucramiento o aceptación hacia los hijos es bajo pero se muestran un alto grado de control y supervisión. Estos padres se inclinan por tener el control del comportamiento de sus hijos. Límites estrictos, obediencia, respeto, orden y poca comunicación son característicos del ambiente que se viven con padres autoritarios. Se presenta de forma común la poca atención de los padres hacia sus

hijos y muy rara vez los cuestionamientos por parte de los niños tienen cabida en el ejercicio de las reglas, los lineamientos establecidos simplemente se deben de asumir. Los niños que son criados bajo este estilo parental son carentes de espontaneidad, curiosidad, originalidad, son muy obedientes y suelen ser dominados por sus pares Baurmind (en Vallejo, Osorno y Mazadiego, 2008).

Las medidas de castigo o punitivas son importantes para este estilo de padre pues según él, es el modelamiento de voluntad más efectivo. Considera que la obediencia es una virtud que debe encontrarse presente en el hijo. Se esfuerza por limitar el desarrollo de la autonomía del niño y encomienda labores en el hogar para fomentar la costumbre al trabajo. Este padre cuida celosamente que el orden se mantenga dentro del hogar (Rossman y Rea, 2005 en Vázquez, 2013).

b) Democrático: este estilo corresponde a los padres cuyos niveles de involucramiento o aceptación y control o supervisión son altos. Estos padres esperan que sus hijos manifiesten conductas maduras, tienen reglas firmes, es común que se usen órdenes y sanciones cuando debe ser necesario. Son padres que están abiertos al análisis e incitan a que sus hijos sean independientes e individuales. Aunque estos padres son exigentes, también atienden las necesidades de sus hijos, es común que persuadan a sus hijos con argumentos convincentes y dando la oportunidad de réplica (Baurmind en Vallejo, Osorno, y Mazadiego, 2008).

Según Musito (en Climent, 2009), la estabilidad psíquica y social, así como el autocontrol y la alta autoestima, están relacionadas con los hijos de padres democráticos, ya que éstos trazan los límites de forma no arbitraria.

En definitiva, este padre refuerza las cualidades de su hijo y dirige de manera guiada 


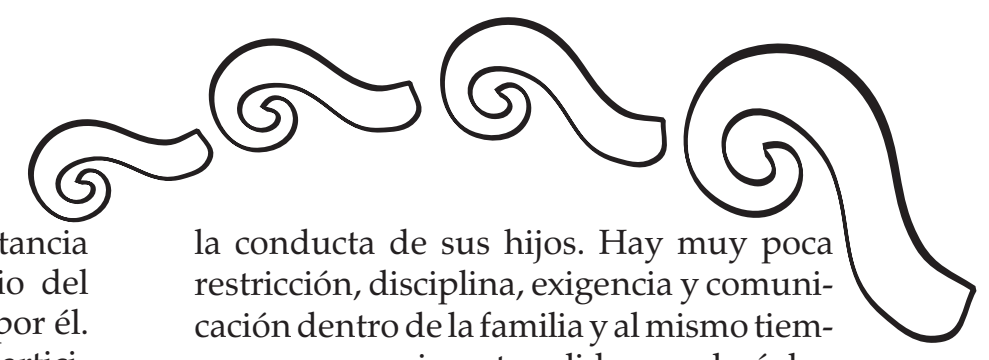

sus actividades, remarca la importancia del alcance de objetivos por medio del esfuerzo propio pero acompañado por él. Aunque hace del niño una persona participativa, con decisiones y sensibilidad, es importante mencionar que no pierde la perspectiva que le brinda la adultez sin ser indulgente, brinda al niño un estatus que refuerza su seguridad y proporciona la posibilidad de una madurez instaurada de manera correcta, ya que toma en cuenta la argumentación del niño y lo responsabiliza de su toma de decisiones sin ser autoritario (Quintana, 1993).

c) Estilo permisivo: son aquellos padres que presentan altos niveles de involucramiento o aceptación y bajos niveles de control o supervisión. Estos padres suelen ser tolerantes y aceptan los impulsos que llegan a tener sus hijos. Los castigos son pocos y en ocasiones no existen, esto hace que los niños experimenten una clara autorregulación. Baurmind (en Vallejo, Osorno y Mazadiego, 2008) menciona que estos padres no controlan ni ejercen la autoridad que deberían, mantienen la esperanza en la razón del niño. Los niños que son criados bajo este estilo suelen tener un limitado control de impulsos y poca autoconfianza.

Este padre permite que el niño regule por sí solo las actividades que lleva a cabo sin ejercer control o marcar limites definidos, su involucración es poca o nula. Dentro de este estilo, se utilizan métodos manipulativos para el control del niño, sin embargo el uso de la razón no le atañe poder sobre las decisiones del niño. No existen métodos coercitivos en este estilo y asumen que sus hijos son ya personas maduras y capaces de desarrollarse sin guías fijas (Vallejo, et al., 2002 en Vázquez, 2013).

d) Negligente: los padres que se encuentran dentro de este estilo parental se caracterizan por no exhibir niveles de involucramiento o aceptación y de control hacia po no se experimenta calidez en el núcleo familiar. Los límites no existen y como consecuencia, los niños se rigen bajo sus propias reglas, esto se da porque los padres argumentan que el estrés que viven a diario no les permite dedicar el tiempo y energía necesarios a sus hijos. Este estilo es similar al permisivo, la diferencia radica en la poca atención que reciben los niños, así como la falta de muestras de cariño Baurmind (en Vallejo, Osorno y Mazadiego, 2008).

Igualmente, Gracia, Lila y García (2011) mencionan que las dimensiones de los estilos parentales tienen parentesco con actitudes como control y afecto, coerción e imposición, aceptación e implicación. Los estudios revisados para este trabajo nombran como estilo parental ideal al democrático y también como el más asociado a un nivel de adaptación psicosocial, aunque también existen estudios que afirman que este estilo no se adapta de manera completa a todos los niños y la instauración de éste depende del tipo de cultura en la que la familia se desarrolle.

\section{Método}

\section{Sujetos}

Constó de 144 niños entre mujeres y varones, los cuales comprendieron un rango de edad de 9 a 12 años de edad, los sujetos son estudiantes regulares de educación primaria del colegio "Jean Piaget", los cuales se encuentran en la cuidad de Actopan, Hidalgo. Asimismo, las madres de estos niños $(n=144)$ formaron parte de la población a evaluar.

\section{Instrumentos}

Para realizar la presente investigación se utilizaron dos pruebas para verificar la correlación entre los estilos parentales y la autoestima en los niños. 


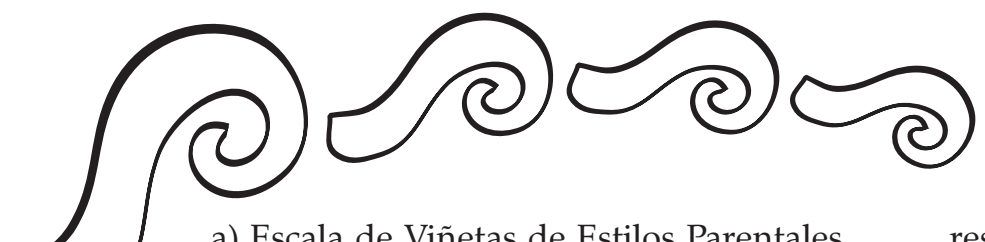

a) Escala de Viñetas de Estilos Parentales (EVEP) (Vázquez, 2013). Este instrumento consta de 22 viñetas que ejemplifican situaciones de la vida cotidiana dentro del contexto familiar y la forma de actuar de los padres con sus hijos. La confiabilidad y validez de la prueba fue realizada mediante regresiones lineales, vinculando el estilo parental con las calificaciones de sus hijos, con lo que se obtuvo una validez predictiva teórica en un modelo de tres pasos (58\% de la varianza explicada).

b) A-EP Cuestionario para la Evaluación de la Autoestima en niños de Primaria (Ramos, Giménez, Muñoz-Adell y Lapaz, 2003). Realizado por Ramos, Gimenez, Muñoz-Adell y Lapaz en el 2003. Dicho cuestionario se valida con una muestra total de $N=1.096$. Este instrumento consta de 12 ítems que describen situaciones por las cuales el niño puede atravesar en su contexto familiar, escolar, social o personal. Estas son viñetas ilustradas para facilitar el proceso de aplicación ya sea de forma grupal o individual. En dichas situaciones el niño debe de contestar eligiendo una de las siguientes opciones: Sí, A veces, No. Mediante la aplicación de un análisis factorial se obtuvieron cinco facto-

res: F1, F2, F3, F4 y F5, donde los cinco factores extraídos explican una varianza total de $50 \%$. Una vez llevada a cabo la prueba " $t$ de student" y "Chi 2", la confiabilidad alcanzada fue de .745 de alfa de Cronbach.

Ambos instrumentos son impresos en hojas de papel para ser contestados con lápiz de grafito y su aplicación es posible de forma grupal e individual.

\section{Resultados}

Para la presente investigación se utilizaron dos muestras poblacionales, niños de 9 a 12 años de edad y sus madres. La población infantil arrojó resultados descriptivos acerca de los 144 niños, de los cuales el $60.4 \%$ son de mujeres y el $30.6 \%$ corresponde a varones; asimismo, el $38.2 \%, 30.6 \%, 24.3 \%$ y 6.9 a los 9 , 10,11 y 12 años respectivamente. Con respecto al grado escolar del cual forman parte los niños, los datos arrojan que la cantidad de 28 , 42,45 y 29 alumnos corresponden a 3ro, 4to, 5to y 6to año de primaria. En el gráfico siguiente se observa el nivel de autoestima que los niños presentan de acuerdo a la prueba A-EP Cuestionario para la Evaluación de la Autoestima en niños de Primaria.

Figura 1 Nivel de autoestima de los alumnos en relación con la frecuencia

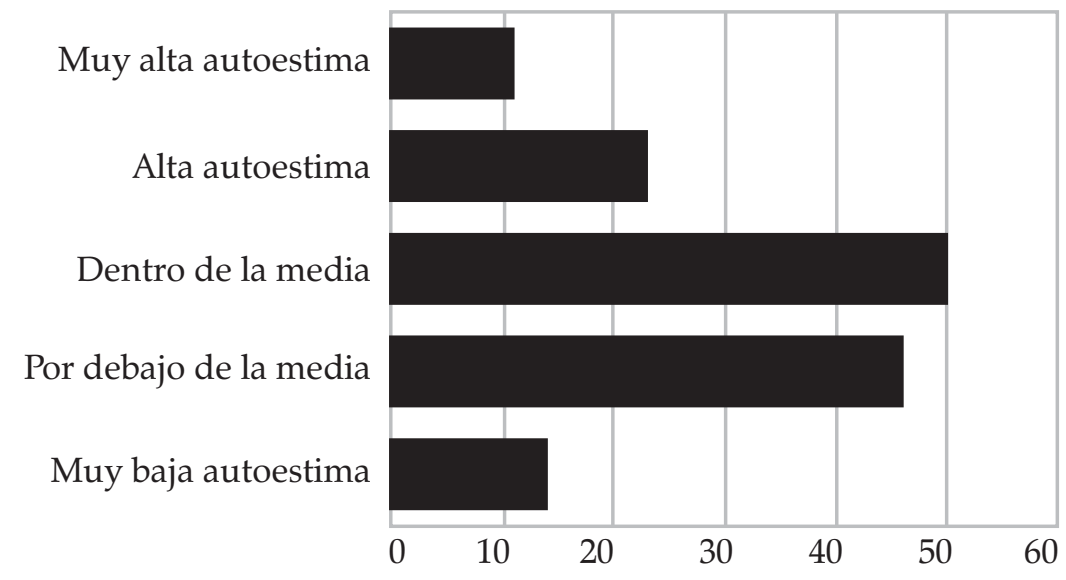

- Nivel de autoestima con relación con la frecuencia 


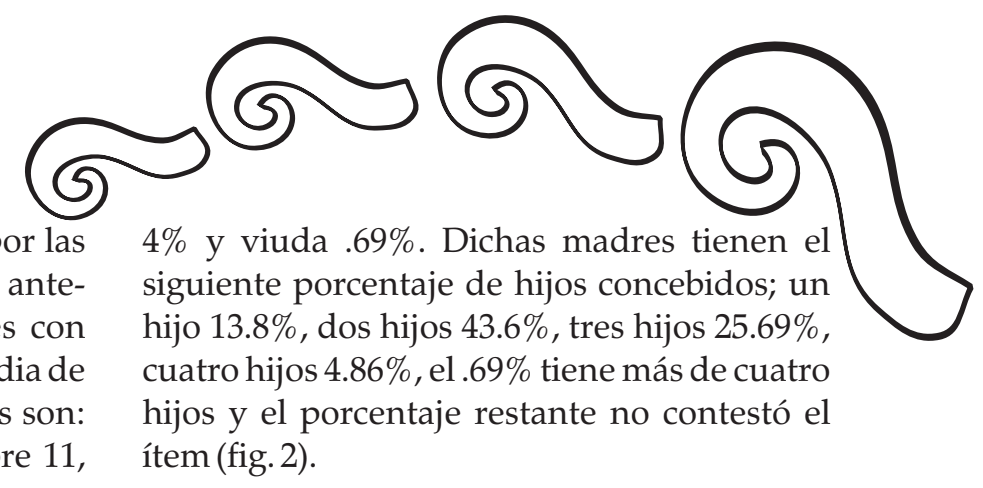

En relación a la muestra conformada por las madres de los alumnos que aplicaron anteriormente, se obtiene que son mujeres con edades de los 27 hasta los 54 con una media de 34.2 años de edad, cuyos estados civiles son: casadas $70 \%$, solteras $4 \%$, en unión libre 11 ,

$4 \%$ y viuda $.69 \%$. Dichas madres tienen el siguiente porcentaje de hijos concebidos; un hijo $13.8 \%$, dos hijos $43.6 \%$, tres hijos $25.69 \%$, cuatro hijos $4.86 \%$, el $.69 \%$ tiene más de cuatro ítem (fig. 2).

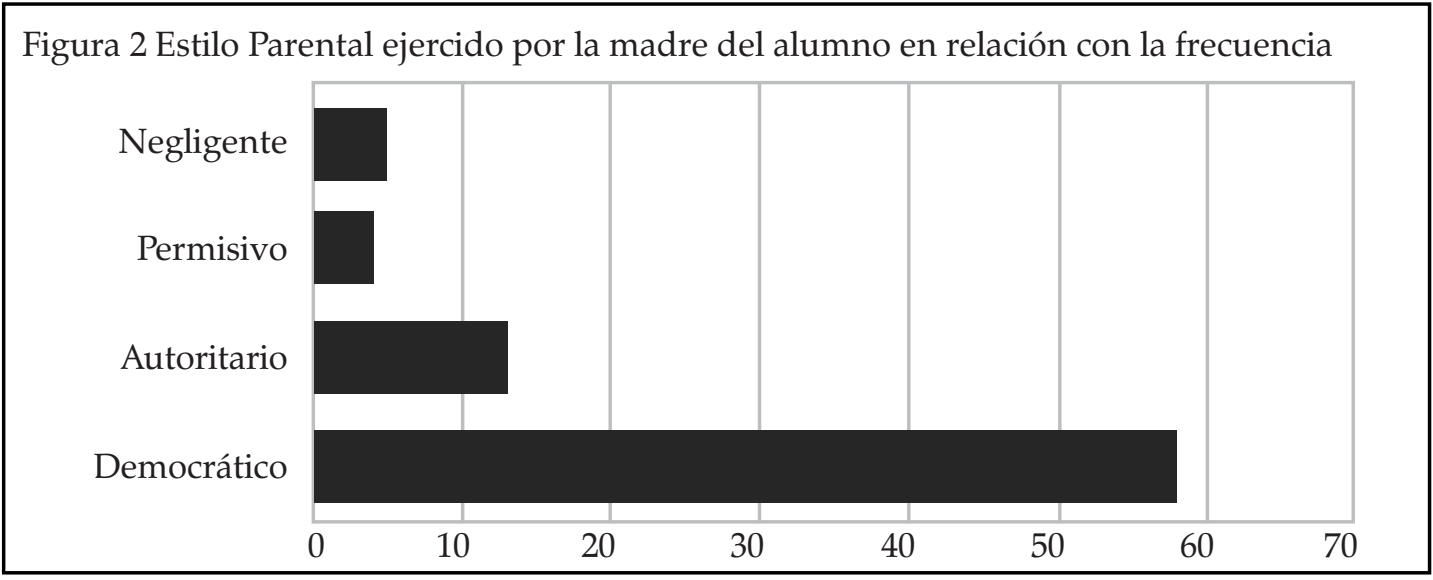

El análisis inferencial que fue llevado a cabo tuvo como finalidad establecer si existe una relación entre el nivel de autoestima que desarrolla el niño y el estilo parental que ejerce la madre sobre él, así como otros factores que pudieran estar relacionados con el estilo parental como lo es el sexo del hijo. Para determinar si el sexo de los hijos influye en el estilo parental que ejerce la madre, así como en la autoestima que presenta, se utilizó un análisis para muestras independientes de $t$, las cuales se muestran a continuación. Donde $p$ $\geqslant .05$ y $\mathrm{p}=.211$. De igual forma en el análisis se encontró que, $\mathrm{p} \geqslant .05$ y $\mathrm{p}=.262$ (tabla 1$)$.

Tabla 1 Prueba t para muestras independientes

\begin{tabular}{lccccc}
\cline { 2 - 6 } & \multicolumn{2}{c}{ Mujeres } & \multicolumn{3}{c}{ Hombres } \\
\cline { 2 - 6 } Estilo Parental & $\mathrm{M}$ & $\mathrm{DE}$ & $\mathrm{M}$ & $\mathrm{DE}$ & $\mathrm{gl}(144)$ \\
\cline { 2 - 6 } & 3.25 & 2.339 & 3.77 & 2.244 & 142 \\
\hline Autoestima & 26.80 & 4.121 & 26.75 & 3.69 & 141 \\
\hline
\end{tabular}

Asimismo, fue realizado un estadístico de contraste mediante $\mathrm{X} 2$ para encontrar la significación de la relación entre estilos parenta-

Tabla 2 Estadístico de contraste

\begin{tabular}{lll}
\cline { 2 - 3 } & Estilo Parental & Nivel de Autoestima \\
\hline Chi-cuadrada & 74.500 & 44.542 \\
gl & 5 & 4 \\
Sig. asintot & .000 & .000 \\
\hline
\end{tabular}

les ejercidos por las madres y la autoestima presentada por sus hijos e hijas (Tabla 2). 


\section{Fuentes de consulta}

Aguirre, A. (2011). Los estilos parentales y la personalidad antisocial: una aproximación al caso de los secuestradores. Tesina de Licenciatura. Universidad Autónoma de México: México.

Andrade, Palos, Delgado y Palacios. (2006). Escala de Estilos Parentales.

Barrón, B. (20012). Los estilos de crianza y su vínculo con el rendimiento académico. Tesis de Licenciatura, Universidad Nacional Autónoma de México, México D.F.

Borja, I. y Romero, E. (2009). Estilos Parentales Percibidos, Psicopatología y Personalidad en la Adolescencia. Revista de Psicopatologia y psicología Clinica, 14 (2), 63-77.

Carreño, P. (2013). Relación entre ambiente familiar y la conducta antisocial en un grupo de adolescentes en conflicto con la ley en un centro tutelar del estado de Morelos. Tesis de Licenciatura. Universidad Nacional Autónoma de México: México.

Casarín, A., Osornio, R., y Mazadiego, T., (2008). Estilos parentales y sintomatología depresiva de una muestra de adolescentes veracruzanos. Revista Latinoamericana de Psicología, 13 (1), 89 110.

Delgado, A., Parras, A., Sánchez - Queija, I. y López, F (2007). Estilos educativos materno y paterno. Evaluación y relación con el ajuste adolescente. Revista de Psicología. (5). 6, 45-97.

Díaz-Guerrero, R. (2003). Bajo las garras de la cultura. La Psicología del Mexicano 2. México: Trillas.

Díaz, P. (2007). Importancia de los amigos y de los padres en el rendimiento escolar. Grupo Editor Latinoamericano, Buenos Aires.

Gómez, J., Luengo, M., Otero, J., Romero, E. y Tavares, E. (1999). Análisis de Ítems para la evaluación de conducta antisocial: un estudio transcultural. RIDEP, 1.

Hernández, D. y Sierra, L. (2012). Los estilos parentales y su relación con la autoestima, depresión y agresión en la adolescencia temprana. Tesis de Licenciatura. Universidad Nacional Autónoma de México: México.

Lemus, R. (2005). Estilos Parentales y su relación con las capacidades académicas y los problemas de conducta en adolescentes. Revista Latinoamericana de Educación 4 (63), 15-58.

Merino, C., y Arndt, S. (2004). Análisis factorial confirmatorio de la escala de estilos de crianza. Revista de Psicología. (3). 2,26-52.

Rodrigo, J. Ma., Mariquez, Ma. L., García, M., Mendoza, R., Rubio, A., Martínez, A. y Martín, J. C. (2004). Relaciones padres - hijos y estilos de vida en la adolescencia. Psicothema 16 (002), 203 -210 .

Salazar, N., López, L y Romero, M. (2005). El rendimiento académico, un fenómeno de múltiples relaciones y complejidades. Revista científica electrónica de psicología,(1).

Sastre, S. (2008). Niños con altas capacidades y su funcionamiento cognitivo diferencial. México: Fondo de Cultura.

Vázquez, S. C. (2013). Diseño y validación de una escala de viñetas para evaluar estilos parentales en la infancia. Tesis de Doctorado. Universidad Iberoamericana: México.

Vera, J. (2012). Conducta antisocial: Asociación con psicopatología en niños y adolescentes. Tesis de Doctorado. Universidad Autónoma de Barcelona: España.
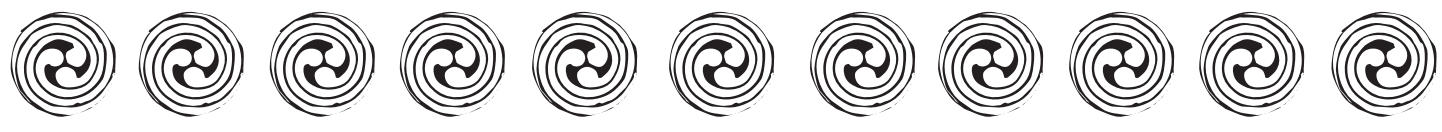

๑2 $42 @$ 